observations also show a similarity in behaviour between gas bubbles in liquid and fluidized solids systems even though the actual mechanism of bubble formation in these two systems must obviously be quite different.

P. D. BLoore

J. S. M. BotTerill

Department of Chemical Engineering,

The University,

Birmingham 15.

1 Davidson, J. F., Paul, R. C., Smith, M. J. S., and Duxbury, H. A. Trans. Inst."Chem. Eng., 37 , 323 (1959).

'Davies, R. M., and Taylor, G., Proc. Roy. Soc., A, 200, 375 (1950).

${ }^{3}$ Calderbank, P. H., Trans. Inst. Chem. Eng., 34, 79 (1956).

\section{Nucleation in Agitated Solutions}

Mechanical disturbances are known to speed the onset of nucleation in supersaturated solutions. Early workers ${ }^{1}$ demonstrated that severe mechanical shock could induce nucleation in metastable solutions at temperatures well above the so-called 'metastable limit', and more recent work ${ }^{2}$ has shown that mechanical stirring can cause rapid nucleation in unseeded solutions which will not normally form nuclei under quiescent conditions. Published work to date has not given any indication of the effect of varying the intensity of agitation on the tendency of an unseeded solution to form nuclei under any given set of conditions, but it is generally assumed that more vigorous agitation increases this tendency.

Investigations carried out in these laboratories on nucleation and growth conditions in a pilot-scale Oslo crystallizer operating with unseeded solutions of ammonium dihydrogen phosphate have confirmed that the critical supersaturation, defined here as the degree of supersaturation at which nuclei are first observed in a solution being cooled at a constant rate, is dependent on the rate of circulation of the liquor, that is, on the degree of turbulence. Less supersaturation is required for nucleation to occur at the higher rates of flow.

It was observed, however, that at low rates of circulation, represented by Reynolds numbers of about $700-1,400$ in a column of 3 in. diameter, the degree of supersaturation necessary for nucleation was not apparently reduced by increasing the turbulence. Unfortunately, variations in the value of the critical supersaturation were here of the same order as the experimental error. Accordingly, recourse was made to a smaller apparatus where conditions were more easily controlled. A glass $T$-stirrer was used in a small baffled beaker. The solutions of ammonium dihydrogen phosphate were covered with a thick layer of liquid paraffin and cured for about $30 \mathrm{~min}$. at $10 \mathrm{deg}$. C. above their saturation temperatures before each run. Accidental inoculation by dust particles was thereby largely eliminated and reproducible results were obtained.

Whereas gentle stirring causes nucleation in solutions which are otherwise stable, and sufficiently vigorous stirring creates an even greater tendency to form nuclei, the transition may not be continuous; no simple relationship between the critical supersaturation and the intensity of agitation has been found. In general, a plot of critical supersaturation against rate of agitation is not a single smooth curve ; in fact, a portion of the curve may have a reversed slope (see Fig. 1) indicating a region where an increase in the agitation reduces the tendency to nucleate. So

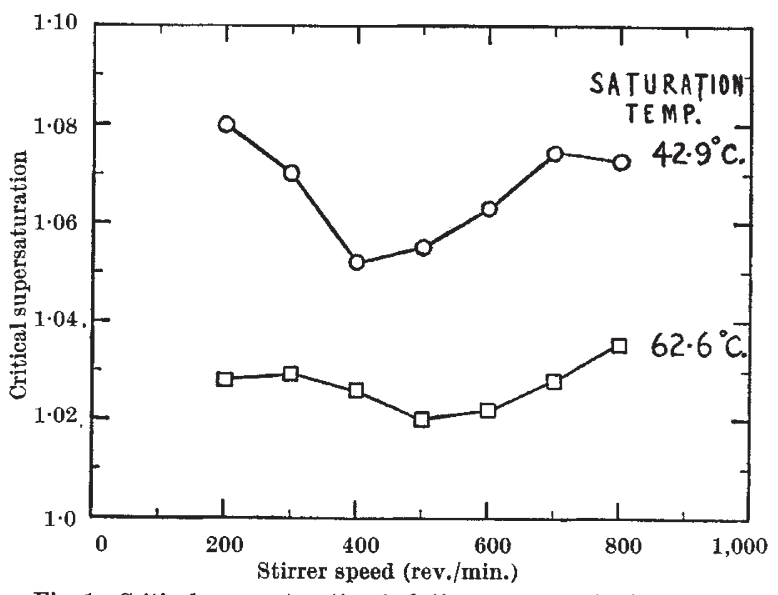

Fig. 1. Critical supersaturation (solution concentration/saturation concentration) versus stirrer speed for aqueous solutions of ammonium dihydrogen phosphate

far as is known, this is the first experimental evidence of such a phenomenon.

Nucleation in liquids has hitherto been considered as consisting of two stages : a diffusional transport step which is aided by agitation, and a surface reaction step when growth of the sub-nucleus occurs, the latter not being influenced by agitation. It would now appear that a third effect may also operate, which impedes the growth of, or disrupts, the sub-nuclei, and that this effect may predominate in certain circumstances.

The work, which so far has been confined to aqueous solutions of ammonium dihydrogen phosphate with saturation temperatures in the range $40-70^{\circ} \mathrm{C}$., is being extended to cover a wider range of concentration and other solutes, to determine whether this effect is of general occurrence or specific to a limited range of conditions, and to attempt a theoretical explanation.

One of us (K. D. R.) is grateful to the Department of Scientific and Industrial Research for a studentship, during which this work is being carried out.

J. W. Mulein

K. D. RAVEN

Department of Chemical Engineering, University College,

$$
\text { London. }
$$

${ }^{1}$ Young S. W., et al. J. A mer. Chem. Soc, 33, 148 (1911); 35, 1067 (1913). Earl of Berkeley, Phil. Mag., (6) 24, 254 (1912).

${ }^{2}$ Matusevich, L. N., J. App. Chem. U.S.S.R. (in English) (2), 27, 139 (1954).

\section{Origin of the Word 'Neutron'}

THE word 'neutron' has been attributed to Rutherford by Glasson ${ }^{1}$ and to W. D. Harkins ${ }^{2}$ by Glasstone ${ }^{3}$. It appears likely that it was not used by either Rutherford or Hawkins before about 1920. In both cases, the neutron was a hypothetical combination of a hydrogen nucleus (also called a 'positive electron' or 'proton') and an ordinary negative electron.

I wish to point out that William Sutherland used the word in almost exactly the same sense in two papers published in 1902 , in the first of which there is the passage :

"As it would be convenient to have symbols different from the operational + and - to distinguish the two sorts of electrons, I propose to use the 